\title{
A XKMS-Based Security Framework for Mobile Grid into the XML Web Services
}

\author{
Namje Park, Kiyoung Moon, Jongsu Jang, and Sungwon Sohn \\ Information Security Research division \\ Electronics Telecommunications research Institute (ETRI) \\ 161 Gajeong-Dong, Yuseong-Gu, Daejeon, 305-350, Korea \\ \{namejepark, kymoon, jsjang, swsohn\}@etri.re.kr
}

\begin{abstract}
Mobile Grid, or Wireless Grid Services refers to value-added Grid Service by processing in Mobile environment. Besides Mobile Internet the traditional Internet computing is experiencing a conceptual shift from ClientServer model to Grid and Peer-to-Peer computing models. As these trends, Mobile Internet and the Grid, are likely to find each other the resource constraints that Wireless devices pose today affect the level of interoperability between them. The goal of this paper is to investigate how well the most limited Wireless devices can make use of Grid Security Services. This paper describes a novel security approach on Mobile Grid Services to validate certificate based on current Mobile XML Web Services Platform environment using XKMS (XML Key Management Specification) and SAML (Security Assertion Markup Language), XACML (extensible Access Control Markup Language) in XML Security mechanism.
\end{abstract}

\section{Introduction}

Grid Computing emerges as a technology for coordinated large-scale resource sharing and problem solving among many autonomous groups. In Grid's resource model, the resource sharing relationships among virtual organizations are dynamic. However, Grid requires a stable quality of service provided by virtual organizations and the changing of sharing relationship can never happen frequently. This model works for a conventional distributed environment but is challenged in the highly variational wireless mobile environment[3].

Besides Mobile Internet the traditional Internet computing is experiencing a conceptual shift from Client-Server model to Grid and Peer-to-Peer computing models. As these trends, Mobile Internet and the Grid, are likely to find each other the resource constraints that Wireless devices pose today affect the level of interoperability between them[2].

Grid is the umbrella that covers many of today's distributed computing technologies. Grid technology attempts to support flexible, secure, coordinated information sharing among dynamic collections of individuals, institutions, and resources. This includes data sharing but also access to computers, software and devices required by computation and data-rich collaborative problem solving. So far the use of Grid services has required a modern workstation, specialized software 
installed locally and expert intervention. In the future these requirements should diminish considerably. One reason is the emergence of Grid Portals as gateways to the Grid. Another reason is the 'Web Service' boom in the industry. The use of XML as a network protocol and an integration tool will ensure that future Grid peer could be a simple wireless device[2,3].

Furthermore, open Mobile Grid service infrastructure will extend use of the Grid technology or services up to business area using Web Services technology. Therefore differential resource access is a necessary operation for users to share their resources securely and willingly. Therefore, this paper describes a novel security approach on open Mobile Grid service to validate certificate based on current Mobile Grid environment using XKMS (XML Key Management Specification) and SAML (Security Assertion Markup Language), XACML (eXtensible Access Control Markup Language) in XML(eXtensible Markup Language) security mechanism.

This paper is organized as follows. First we investigate related work on Grid and mobile web service, XML web service security. Then we propose a design of security system platform for open mobile Grid service and explain experimented XKMS model for certificate validation service. Finally, we explain function of system and then we conclude this paper.

\section{Mobile XML Web Services}

A mobile XML web service can feature one of the following architectures: wireless portal network, wireless extended Internet, or wireless ad hoc network.

In a wireless portal network, the wireless information devices connect to the Internet backend services through portal entry points. The portal creates a "walled garden" and controls access to Internet contents. Wireless portal networks support widely deployed thin-client wireless technology, such as WAP (Wireless Application Protocol). The portal receives the message, checks the user's privilege, and then translates the request to a SOAP (Simple Object Access Protocol) message or an XML-RPC call to an appropriate partner web service. The web service replies, and the portal translate the response back to a WML (Wireless Markup Language) document. The portal sends the WML document back to the wireless device for display. In this way, the portal works as a proxy for wireless users. The portal operator provides user authorization and management services. Many partner vendors can provide real application web services under the ASP (Application Service Provider) model.

Wireless extended Internet is the wired Internet's expansion to wireless devices. Wireless information devices can have their own IP addresses (through Internet Protocol 6) and full network functionalities. Those devices usually run smart, fat clients that interact with multiple backend services simultaneously and store/process application data on the device. Smart devices support sophisticated user interfaces, offline processing, and automatic transactions. They can also implement flexible, application-specific security policies. Like the Internet itself, the wireless extended Internet architecture is decentralized and eliminates any single point of failure. However, as you will see later, centralized web services hubs are still required to support advanced security schemes and user interfaces. Unlike the portal architecture, the hubs themselves can be decentralized. Different vendors can provide similar hub 


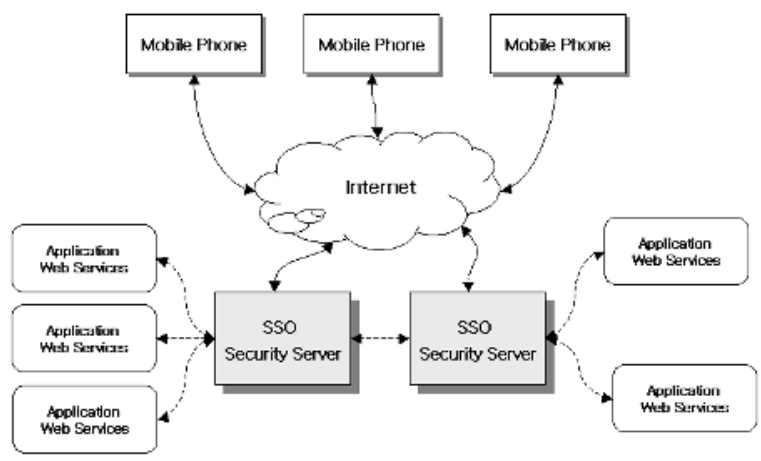

Fig. 1. Mobile Web Services Architecture

services that can interoperate with each other. Fig. 1 shows a topography diagram for such networks.

The extended wireless Internet architectures blended with decentralized hub web services will provide the foundation for future wireless web services applications, an approach we focus on throughout this article. Since most of the supporting technologies are just emerging, many challenges prevail.

The wireless ad hoc networks allow wireless devices to become servers to peers. Wireless peers can provide content, network traffic routing, and many other services. The ad hoc network truly leverages wireless networks' dynamic nature. However, because wireless peer-to-peer technology is still embryonic, its many performance and security issues must be solved before it can be widely used.

\section{Security Framework for Providing Secure Open Mobile Grid}

Web services can be used to provide mobile security solutions by standardizing and integrating leading security solutions using XML messaging. XML messaging is referred to as the leading choice for a wireless communication protocol and there are security protocols for mobile applications based upon it. Among them are the follows. SAML is a protocol to transport authentication and authorization information in an XML message. It could be used to provide single sign on web services. XML signatures define how to digitally sign part or all of an XML document to guarantee data integrity. The public key distributed with XML signatures can be wrapped in XKMS formats. XML encryption allows applications to encrypt part or all of an XML document using references to pre-agreed symmetric keys. The WS-Security, endorsed by IBM and Microsoft, is a complete solution to provide security to web services. It is based on XML signatures, XML encryption, and an authentication and authorization scheme similar to SAML. When a mobile device client requests access to a back-end application, it sends authentication information to the issuing authority. The issuing authority can then send a positive or negative authentication assertion depending upon the credentials presented by the mobile device client. While the user still has a session with the mobile applications, the issuing authority can use the earlier reference to send an authentication assertion stating that the user was, in fact, authenticated by a 


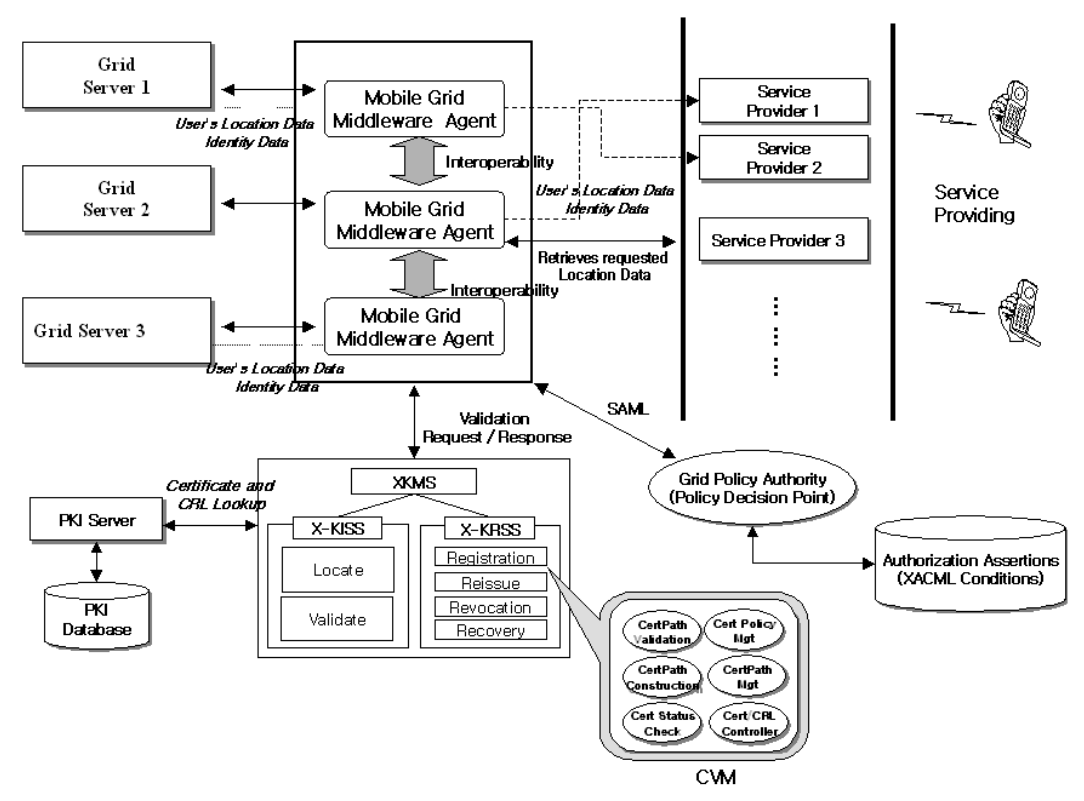

Fig. 2. Security Service Model for Open Mobile Grid Middleware

particular method at a specific time. As mentioned earlier, location-based authentication can be done at regular time intervals, which means that the issuing authority gives out location-based assertions periodically as long as the user credentials make for a positive authentication.

CVM (Certificate Validation Module) in XKMS system perform path validation on a certificate chain according to the local policy and with local PKI (Public Key Infrastructure) facilities, such as certificate revocation (CRLs) or through an OCSP (Online Certificates Status Protocol). In the CVM, a number of protocols (OCSP, SCVP, and LDAP) are used for the service of certificate validation. For processing the XML client request, certificate validation service from OCSP, LDAP (Lightweight Directory Access Protocol), SCVP (Simple Certificate Validation Protocol) protocols in XKMS based on PKI are used. The XKMS client generates an 'XKMS validate' request. This is essentially asking the XKMS server to go and find out the status of the server's certificate. The XKMS server receives this request and performs a series of validation tasks e.g. X.509 certificate path validation. Certificate status is determined. XKMS server replies to client application with status of the server's certificate and application acts accordingly. Using the OCSP protocol, the CVM obtained certificate status information from other OCSP responders or other CVMs. Using the LDAP protocol, the CVM fetched CRL (Certificate Revocation List) from the repository. And CA (Certificate Authority) database connection protocol (CVMP;CVM Protocol) is used for the purpose of that the server obtains real-time certificate status information from CAs. The client uses OCSP and SCVP. With XKMS, all of these functions are performed by the XKMS server component. Thus, there is no need for LDAP, OCSP and other registration functionality in the client application itself. 


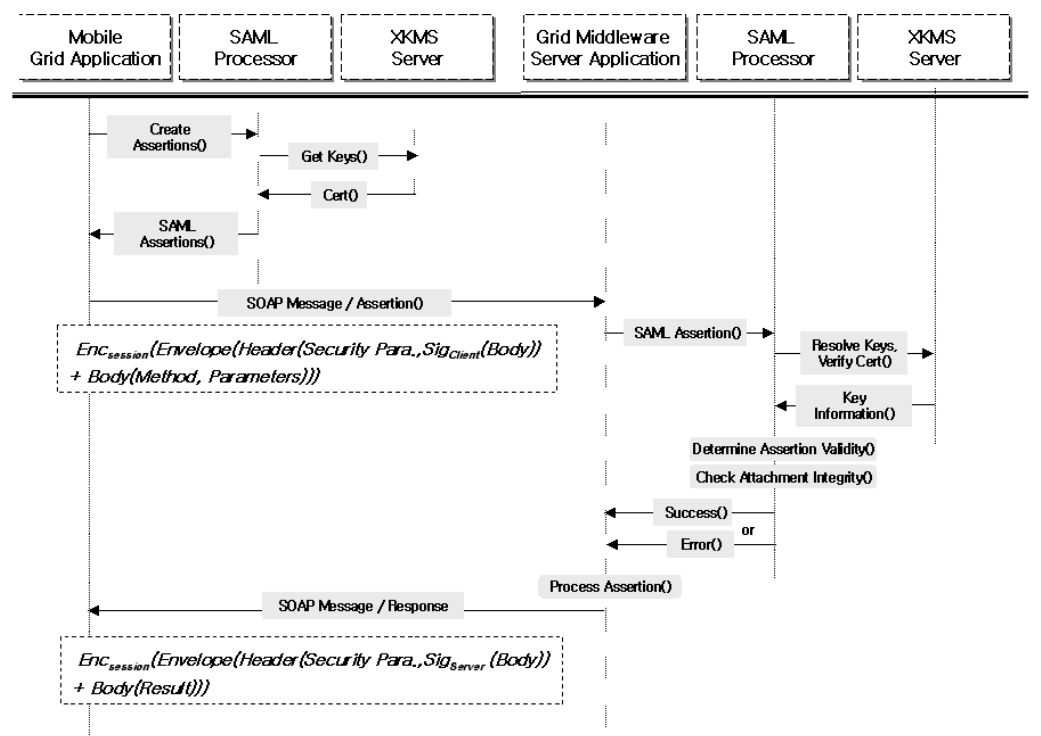

Fig. 3. Security Protocol for Secure Open Mobile Grid Service

\section{Mobile Grid Application Security Protocol}

Three types of principals are involved in our protocol: Mobile Grid application (server/client), SAML processor, and XKMS server (including PKI). Proposed invocation process for secure Mobile Grid security service consists of two parts: initialization protocol and invocation protocol. The initialization protocol is prerequisite for invoking Grid web services securely. Through the initialization protocol, all principals in our protocol set up security environments for their web services, as shown in fig. 3. The flow of setting up security environments is as follows.

The client first registers its information for using web services, and then gets its $\mathrm{id} /$ password that will be used for verifying its identity when it calls web services via secure channel. Then, the client gets SAML assertions and installs security module to configure its security environments and to make a secure SOAP message. It then generates a key pair for digital signature, and registers its public key to a CA.

The client creates a SOAP message, containing authentication information, method information, and XML signature, XML encrypts it, and then sends it to a server. The message is in following form: Enc session $_{\text {(Envelope (Header(SecurityParameters, }}$ $\left.\operatorname{Sig}_{\text {client }}(\operatorname{Body})\right)+\operatorname{Body}($ Method, Parameters $\left.\left.\left.)\right)\right)\right)$, where $\operatorname{Sig}_{x}(y)$ denotes the result of applying $x$ ' s private key function (that is, the signature generation function) to $y$. The protocol shown in fig. 4 shows the use of end-to-end bulk encryption[12]. The security handlers in server receive the message, decrypt it, and translate it by referencing security parameters in the SOAP header. To verify the validity of the SOAP message and authenticity of the client, the server first examines the validity of the client's public key using XKMS. If the public key is valid, the server receives it from CA and verifies the signature. The server invokes web services after completion 


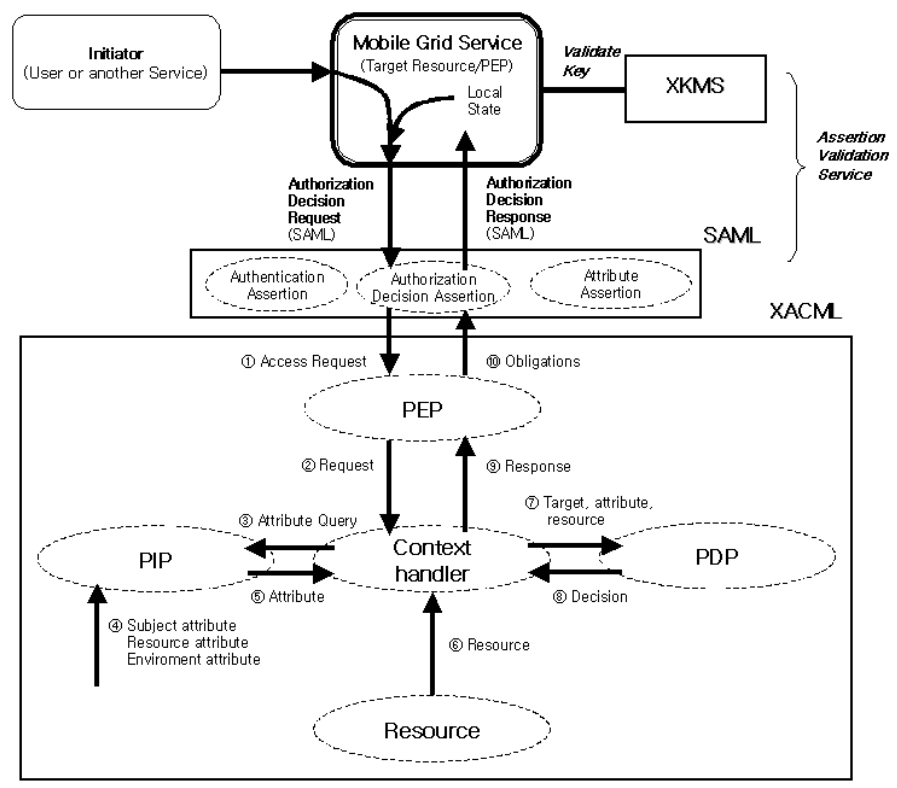

Fig. 4. Security Message Flow using XKMS in Open Mobile Grid Middleware

of examining the security of the SOAP message. It creates a SOAP message, which contains result, signature, and other security parameters. Then, it encrypts the message using a session key and sends it back to the client. Lastly, the client examines the validity of the SOAP message and server, and then receives the result $[14,15]$.

In current Grid service, there is no mechanism of differential resource access. To establish such a security system we are seeking, a standardized policy mechanism is required. We employ the XACML specification to establish the resource policy mechanism that assigns differential policy to each resource (or service). SAML also has the policy mechanism while XACML provides very flexible policy mechanism enough to apply to any resource type. For our implementing model, SAML provides a standardized method to exchange the authentication and authorization information securely by creating assertions from output of XKMS (e.g. assertion validation service in XKMS). XACML replaces the policy part of SAML as shown in fig 4.

Once the three assertions are created and sent to the protected resource, there is no more verification of the authentication and authorization at the visiting site. This, SSO (Single Sign-On), is a main contribution of SAML in distributed security systems.

Fig. 4 shows the flow of SAML and XACML integration for differential resource access. Once assertions are done from secure identification of the PKI trusted service, send the access request to the policy enforcement point (PEP) server (or agent) and send to the context handler. Context handler parses the attribute query and sends it to PIP (policy information point) agent. The PIP gathers subject, resource and environment attributes from local policy file, and the context handler gives the required target resource value, attribute and resource value to PDP (policy decision point) agent. Finally, the PDP decides access possibility and send context handler so that PEP agent allow or deny the request[10,13]. 


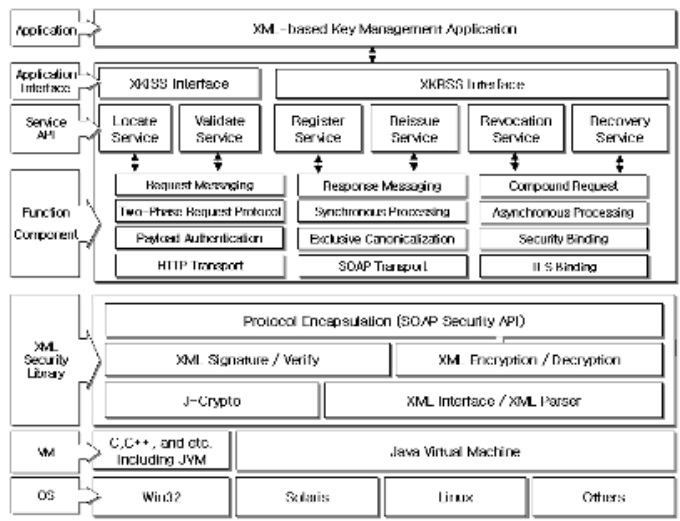

Fig. 5. Package Library Architecture of XKMS Server System based on CAPI

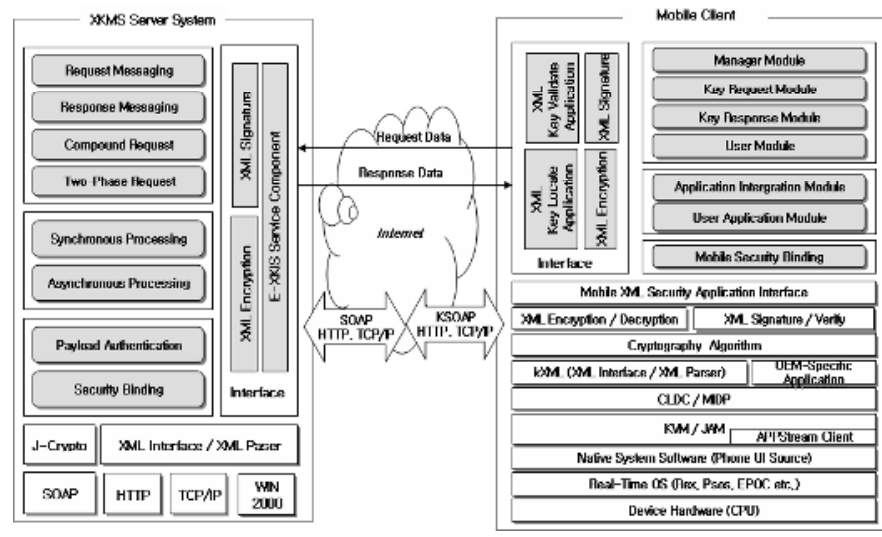

Fig. 6. Testbed Configuration of XKMS Component for Open Grid

\section{Design of Mobile Grid Application Key Management Using XKMS}

XKMS has been implemented based on the design described in previous section. Package library architecture of XKMS based on CAPI (Cryptographic Application Programming Interface) is illustrated in fig. 5.

Components of the XKMS are XML security library, service components API, application program. Although XKMS service component is intended to support XML applications, it can also be used in order environments where the same management and deployment benefits are achievable. XKMS has been implemented in Java and it runs on JDK (Java Development Kit) ver. 1.3 or more.

The figure for representing Testbed architecture of XKMS service component is as follows fig. 6. We use Testbed system of windows PC environment to simulate the processing of various service protocols. The protocols have been tested on pentium 3 and pentium 4 PCs. It has been tested on windows 2000 server, windows XP. 
Java 2, Micro Edition (J2ME) is a set of technologies and specifications developed for small devices like smart cards, pagers, mobile phones, and set-top boxes. J2ME uses subset of Java 2, Standard Edition (J2SE) components, like smaller virtual machines and leaner APIs. J2ME has categorized wireless devices and their capabilities into profiles: MIDP, PDA and Personal. MIDP and PDA profiles are targeted for handhelds and Personal profile for networked consumer electronic and embedded devices. As the technology progresses in quantum leaps any strict categorization is under threat to become obsolete. It is already seen that J2ME Personal profile are being used in high-end PDAs such as PocketPCs and Mobile Communicators. We will concentrate on the most limited category of wireless J2ME devices that use Mobile Information Device Profile (MIDP). Applications that these devices understand are Midlets. Typically maximum size of a midlet varies from 30$50 \mathrm{kbs}$ and user can download four to six applications to his mobile phone. Midlet is a JAR-archive conforming to the Midlet content specification[2].

The XKMS server is composed server service component of XKMS platform package. And the message format is based on Specification of W3C (World Wide Web Consortium).

\section{Conclusion}

Mobile Grid services are so attractive that they can cover all walks of life. However, current Grid is growing slower than expected. Many problems like accuracy, privacy, security, customer requirement have to be addressed. It should be understood that there is no single universal solution to Grid.

We propose a novel security approach on open Grid to validate certificate based on current Grid security environment using XKMS and SAML, XACML in XML security. This service model allows a client to offload certificate handling to the server and enable to provide central administration of XKMS polices. In order to obtain timely certificate status information, the server uses several methods such as CRL, OCSP etc. Our approach will be a model for the future security system that offers security of open Grid security.

\section{References}

1. XML Key Management Specification Version 2.0 (W3C Working Draft), April 2003.

2. Miika Tuisku, Wireless Java-enabled MIDP devices as peers in Grid infrastructure, Helsinki Institute of Physics, CERN.

3. Ye Wen, Mobile Grid Major area examination, University of California, 2002.

4. E. Faldella and M.Prandini, A Novel Approach to On-Line Status Authentication of Public Key Certificates, in Proc. the $16^{\text {th }}$ Annual Computer Security Applications Conference, Dec 2000.

5. Y. Elley, A. Anderson, S. Hanna, S. Mullan, R. Perlman and S. Proctor, Building Certification Paths: Forward vs. Reverse, Proc. the Network and Distributed System Security Symposium Conference, 2001.

6. M. Naor and K. Nissim, Certificate Revocation and Certificate Update, IEEE Journal on Selected Areas in Communications, 18 (4) (2000).

7. Jonghyuk Roh et. Al., Certificate Path Construction and Validation in CVS, KICS-Korea IT Forum, 2002. 
8. M. Prandini, Efficient Certificate Status Handling within PKIs: an Application to Public Administration Services, in Proc. the $15^{\text {th }}$ Annual Computer Security Applications Conference, 1999.

9. Donald E, Eastlake, Kitty Niles, Secure XML, Pearson addsion wesley, 2003.

10. Namje Park et. Al., Certificate Validation Scheme of Open Grid Service Usage XKMS, GCC 2003, 2003.

11. Euinam Huh, Jihye Kim, Hyeju Kim, Kiyoung Moon, Policy based on Grid security infrastructure implementation for dirrerential resource access, ISOC 2003, 2003.

12. Yuichi Nakamur, et. Al., Toward the Integration of web services security on enterprise environments, IEEE SAINT '02, 2002.

13. Diana Berbecaru, Antonio Lioy, Towards Simplifying PKI Implementation: Client-Server based Validation of Public Key Certificates, IEEE ISSPIT 2002, pp.277-281.

14. Jose L. Munoz et. Al., Using OCSP to Secure Certificate-Using transactions in MCommerce. LNCS 2846 (2003) 280-292.

15. Sungmin Lee et. Al., TY*SecureWS:An integrated Web Service Security Solution based on java, LNCS 2738 (2003) 186-195. 